\title{
Minimally invasive resection and vertebroplasty for an osteolytic C-1 metastasis of malignant meningioma: case report
}

\author{
Jan-Helge Klingler, MD,1 Marie Therese Krüger, MD, ${ }^{1}$ Evangelos Kogias, MD,1 \\ Stefanie M. Brendecke, MD, ${ }^{2}$ Ulrich Hubbe, MD, ${ }^{1}$ and Christian Scheiwe, MD${ }^{1}$ \\ Departments of ${ }^{1}$ Neurosurgery and ${ }^{2}$ Neuropathology, Freiburg University Medical Center, Freiburg, Germany
}

Malignant meningiomas are a rare but aggressive subset of intracranial meningiomas leading to a very limited life expectancy. The occurrence of spinal metastases in these tumors is an even rarer event. The described patient had an intracranial malignant meningioma and developed a symptomatic osteolytic contrast-enhancing lesion in the left C-1 lateral mass suspicious for metastasis. The authors performed a minimally invasive posterior resection of the lesion with vertebroplasty of $\mathrm{C}-1$. Histopathology verified metastasis of the malignant meningioma. The surgical procedure resulted in prompt and permanent pain reduction until the patient died 18 months later. Given the very limited life expectancy in this case, the authors did not consider occipitocervical fusion because of their desire to preserve the range of motion of the head. Therefore, they suggest minimally invasive tumor resection and vertebroplasty in selected palliative tumor patients.

http://thejns.org/doi/abs/10.3171/2015.1.SPINE141041

KEY WORDS atlas; cement augmentation; cervical spine; minimally invasive; malignant meningioma; metastasis; oncology

I NTRACRANIAL meningiomas originate from arachnoid cap cells and account for $13 \%-26 \%$ of all intracranial tumors. ${ }^{14}$ The World Health Organization (WHO) subclassifies these tumors as benign (Grade I), atypical (Grade II), or anaplastic/malignant (Grade III). ${ }^{13}$ Only $1.0 \%-2.8 \%$ of all meningiomas are classified as malignant, whereas the majority of these lesions are benign. ${ }^{14}$ Malignant meningiomas, however, are a very aggressive subset with a median patient survival of 1.5 years ${ }^{15}$ and reported 5-year survival rates of 32\%-64\%. ${ }^{16}$ The occurrence of malignant intracranial meningiomas with spinal metastases has rarely been reported., $, 6,11,12$

To our knowledge, the featured case is the first description of a patient with an osteolytic C-1 metastasis of an intracranial malignant meningioma that was treated with minimally invasive tumor resection and vertebroplasty of C-1.

\section{Case Report}

Presentation and History

The patient sustained his first focal sensorimotor seizure at the age of 43 years. Magnetic resonance imaging displayed a left frontoparietal convexity meningioma (Fig. $1 \mathrm{~A}$ and $\mathrm{B})$. At the time, however, the patient rejected resection as well as radiation therapy. In the course of the following 2 years, the epilepsy worsened and led to a first grand mal seizure. Magnetic resonance imaging showed progression of the meningioma to $80 \times 62 \times 22 \mathrm{~mm}$ with surrounding edema (Fig. 1C and D). At this time the patient consented to surgery and radiation therapy. During gross-total resection, the meningioma extensively infiltrated the cortex. The preoperatively unimpaired patient experienced a transient slight paresis of the right hand (Grade 

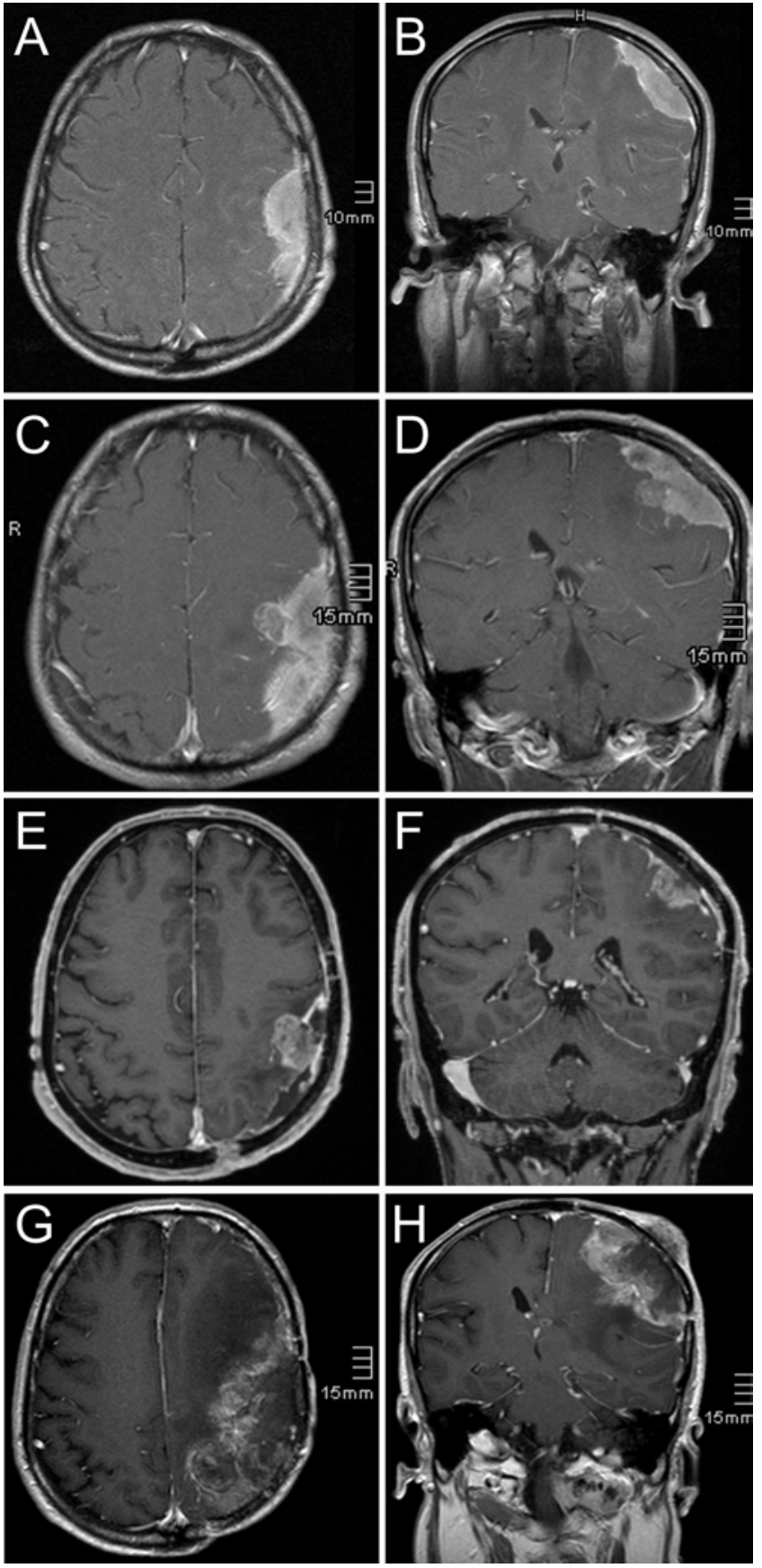

FIG. 1. A and B: First MR images demonstrated evidence of a left frontoparietal convexity meningioma; however, the patient refused treatment at this time. $\mathrm{C}$ and $\mathrm{D}$ : Magnetic resonance images obtained 2 years later, showing tumor progression of the meningioma with surrounding edema. At this time, the patient suffered seizures more frequently and agreed to undergo resection and subsequent radiation therapy. $E$ and F: Magnetic resonance images obtained 9 months after the first surgery and radiation therapy, showing local tumor progression. Epilepsy had again worsened despite anticonvulsive therapy. A second resection was performed. $\mathbf{G}$ and $\mathrm{H}$ : Final MRI follow-up 18 months after the first tumor resection showing progressive intracranial and extracranial tumor growth.
4-5 according to the Medical Research Council grading system) postoperatively. Histopathology showed malignant meningioma (WHO Grade III). Subsequently, the patient received fractionated stereotactic radiation therapy with a cumulative dose of $59.4 \mathrm{~Gy}$.

Nine months after the first surgery, seizures became more frequent again despite anticonvulsive therapy. Magnetic resonance imaging showed local tumor progression (Fig. 1E and F) resulting in a second resection. Additionally, imaging revealed an osteolytic contrast-enhancing lesion in the left C-1 lateral mass (Figs. 2 and 3A) suspicious for metastasis of the known malignant meningioma. The 45-year-old patient now experienced upper neck pain (visual analog scale [VAS] Score 5/10).

\section{Operation}

After obtaining the patient's informed consent, we performed minimally invasive surgery via a posterior approach using a nonexpendable tubular retractor (METRx, Medtronic). After dissection of the muscle fascia via a $2.5-\mathrm{cm}$ skin incision, the dilators were placed on the left posterior arch of C-1 under fluoroscopic image guidance. The initial approach was near the midline to protect the vertebral artery. The nonexpendable tubular retractor with a diameter of $16 \mathrm{~mm}$ was then inserted toward the left posterior arch of C-1 as well as the lateral mass. After partial resection of the lateral posterior arch of C-1 under microscopic vision, the lateral spinal canal with the spinal cord and the lateral mass were reached. After carefully opening the lateral mass with a small diamond drill, the soft, yellowish, slightly glassy tumor could be identified. While mobilizing the extradural lesion inside the lateral mass, a small gap leading to the spinal canal was detected in the cortical bone, which could have been the pathway for metastasis into the lateral mass if CSF dissemination is presumed. ${ }^{2}$ After resection of the tumor, the osteolytic defect of C-1, including the lateral mass, was filled by injecting $1.5 \mathrm{ml}$ of bone cement (polymethylmethacrylate, Vertaplex, Stryker) through the tubular retractor under fluoroscopic image guidance (Fig. 4) without evidence of unintended cement extravasation. The skin was closed with subcutaneous sutures and skin adhesive.

\section{Pathological Findings}

Hematoxylin and eosin staining showed a pleomorphic, solidly growing, highly proliferative meningeal tumor with an overall high cell density, nuclear atypia as well as areas of necrosis, and numerous mitoses, altogether more than 25 in 10 hpf (400-fold magnification; Fig. 5). Immunohistochemical staining with Ki 67 (MIB1) revealed a very high rate of proliferation within the tumor tissue, marking 30\%-40\% of all tumor cells. Most of the meningeal tumor cells displayed immunopositivity for epithelial membrane antigen. These findings led to a diagnosis of malignant meningioma, WHO Grade III.

\section{Postoperative Course}

Postoperatively, the patient had no new neurological deficit and reported an improvement in his neck pain (VAS Score 2/10). He was discharged on the 3rd postoperative day. Since no cervical segments had been fused, his 
J.-H. Klingler et al.
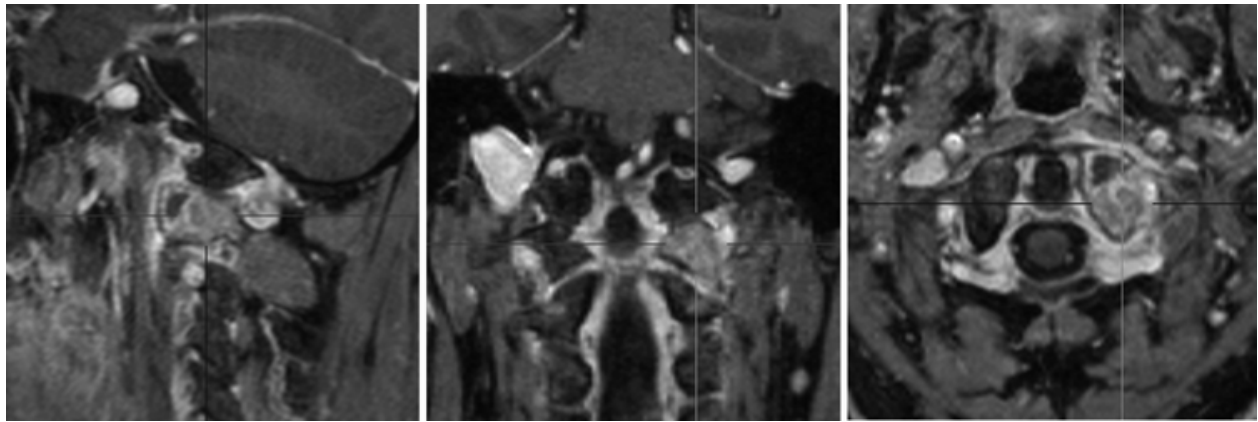

FIG. 2. Preoperative triplanar MR images showing the contrast-enhancing lesion in the left lateral mass of C-1.

head mobility was unimpaired. Postoperative CT showed the intended distribution of bone cement in $\mathrm{C}-1$ without evidence of cement extravasation (Fig. 3B).

Given the pathological findings, subsequent radiation therapy to $\mathrm{C}-1$ was performed (fractionated radiation therapy with a cumulative dose of 35 Gy). Follow-up imaging revealed local tumor progression with bony destruction of the left lateral mass and thus segmental malposition (Cobb angle $10^{\circ}$ ) with deviation of the dens (Fig. 3C). Considering the patient's limited life expectancy and his minor local discomfort, we did not intend to implant corrective instrumentation.

Thirteen months after the first surgery, the patient developed progressive paresis of the right arm and apha-

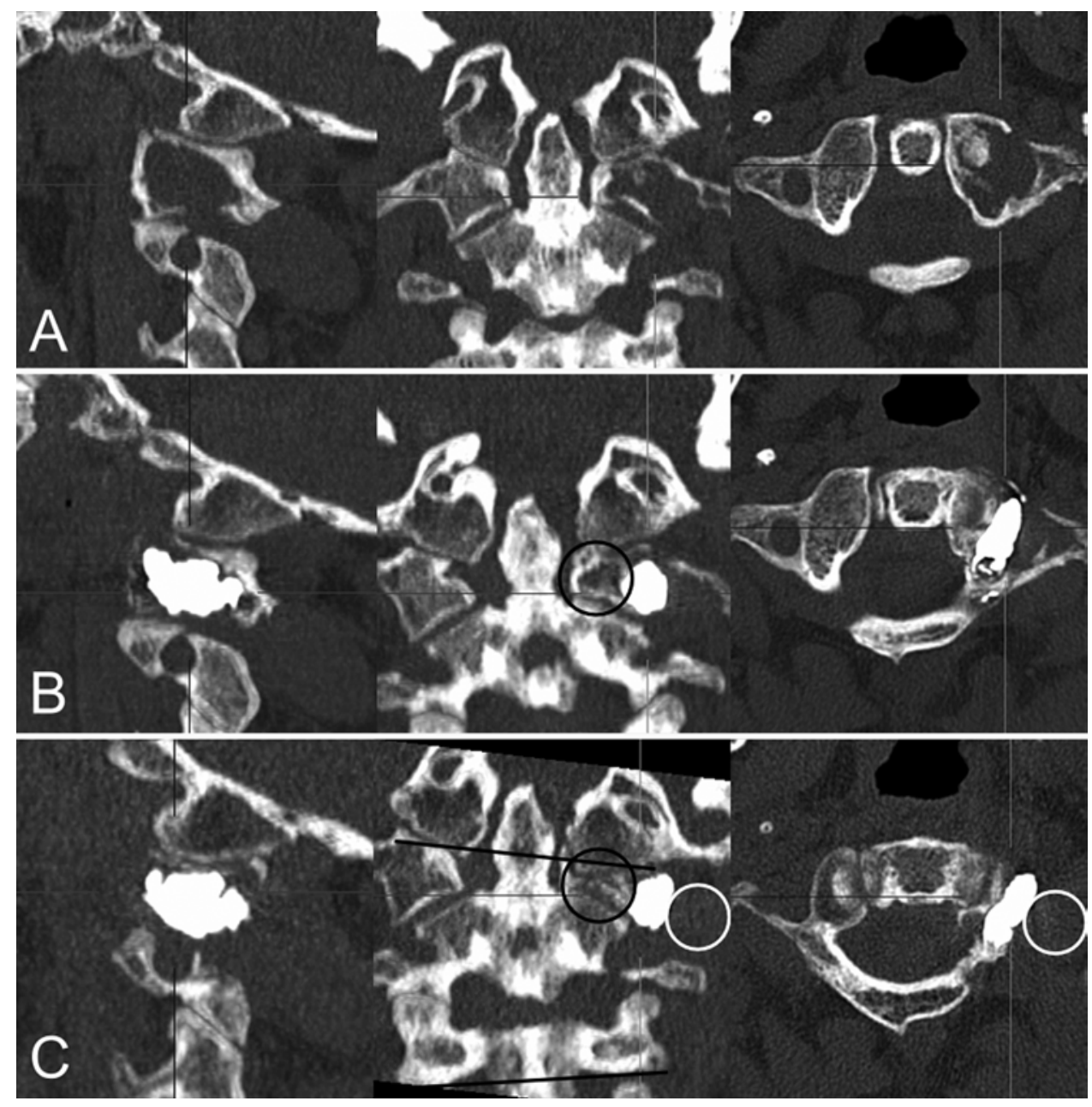

FIG. 3. Triplanar CT scans of the occipitocervical region. A: Preoperative CT showing the osteolytic lesion in the left lateral mass of C-1. B: Postoperative CT showing a desired distribution of bone cement in the left lateral mass of C-1 without evidence of cement extravasation. C: Computed tomography scan obtained 9 months after surgery, showing progressive bony destruction (black circle) as a result of local tumor progression. Note the diminished portion of the medial part of the lateral mass as compared with the immediately postoperative CT (black circles) as well as the progressive bony destruction of the transverse process (white circles) resulting in a segmental deviation of $10^{\circ}$ (black lines). 


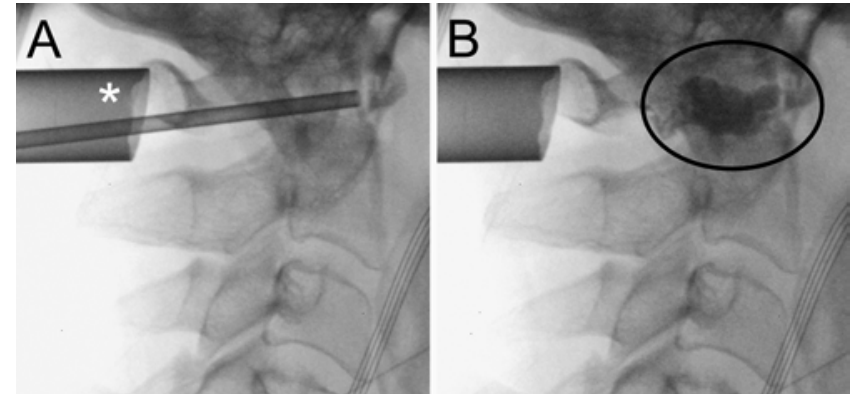

FIG. 4. Intraoperative lateral fluoroscopic images showing the minimally invasive, nonexpendable tubular retractor (asterisk) and the suction device in the lateral mass of $\mathrm{C}-1$ after tumor resection (A). Lateral mass of $\mathrm{C}-1$ (B, circle) after vertebroplasty via the tubular retractor.

sia. Again, the epilepsy worsened. Magnetic resonance imaging depicted intracranial tumor progression with surrounding edema. We performed a third intracranial tumor resection with subsequent adjuvant radiation therapy. Magnetic resonance imaging 5 months later showed further tumor progression with surrounding edema. After 3 resective operations and 2 courses of intracranial radiation therapy, we did not consider repeat surgery to be a reasonable option in this palliative situation. The patient received 4 cycles of temozolomide as nonempirical therapy. The last MRI follow-up 18 months after the first intracranial tumor resection showed progressive intracranial and extracranial tumor growth (Fig. 1G and $\mathrm{H}$ ) and contrast-enhancing local tumor progression around the bone cement in the lateral mass of C-1 (11 months after cervical surgery). Ultimately, the patient died 18 months after tumor resection with vertebroplasty of C-1 and 28 months after the primary intracranial surgery.

\section{Discussion}

Patients with malignant meningioma have a very limited life expectancy with a reported median survival of 1.5 years..$^{15}$ As malignant meningiomas are a rare entity, their metastases to the spine have rarely been reported, $2,6,11,12$ despite an overall reported metastatic rate of $43 \% .^{5}$ Most case reports imply intradural metastases without bony destruction. ${ }^{2,6,11,12}$ In this regard, the presented case is unique because of the exclusively extradural location and concomitant bony destruction. The objective of surgery was to obtain a biopsy specimen and provide stability of the upper cervical spine before administering radiation therapy. Therefore, we considered 3 different surgical procedures: 1) biopsy and occipitocervical fusion with screw-rod instrumentation, 2) biopsy and percutaneous vertebroplasty, and 3) minimally invasive resection and vertebroplasty. Considering the limited life expectancy, ${ }^{15,16}$ we abandoned occipitocervical fusion to preserve head mobility. Percutaneous cervical vertebroplasty would have been another option that has been performed via different approaches in selected patients with osteolytic tumors, ${ }^{1,3,4,7,8,10,17}$ however, this technique is challenging, and violation of the vertebral artery as well as cement extravasation into the vertebral artery or spinal canal can occur. Preoperatively, we assumed a gap in the cortical bone between the tumor and the spinal canal, which implies a higher risk for intra-
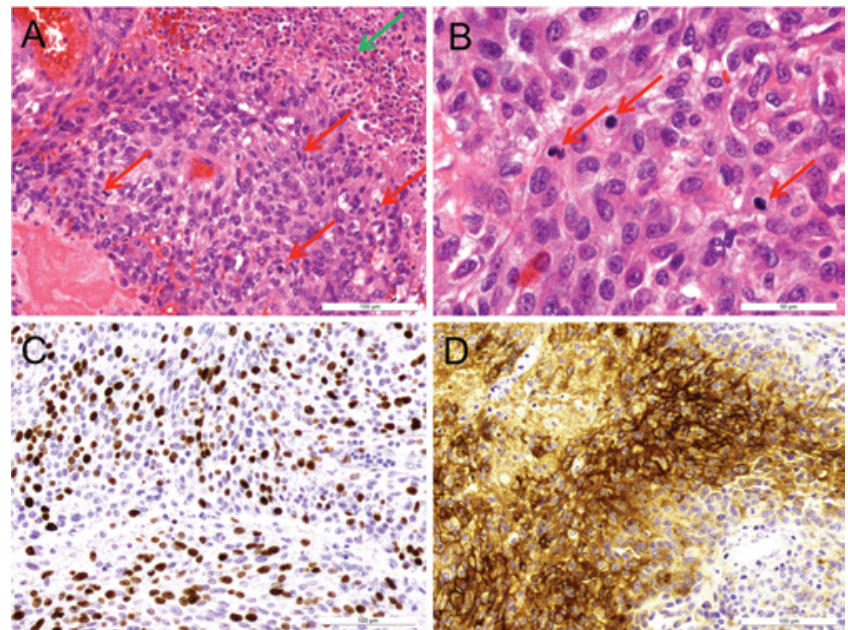

FIG. 5. A and B: Pleomorphic, solidly growing, highly proliferative meningeal tumor with areas of necrosis (green arrow) and numerous mitoses (red arrows). H \& E. C: Immunohistochemical staining with Ki 67 (MIB1) revealed a very high rate of proliferation within the tumor tissue, marking $30 \%-40 \%$ of all tumor cells (brown staining). D: Most of the meningeal tumor cells stained positively for epithelial membrane antigen (brown staining). Bar $=100 \mu \mathrm{m}(\mathrm{A}, \mathrm{C}, \mathrm{D})$ and $50 \mu \mathrm{m}(\mathrm{B})$. Figure is available in color online only.

spinal cement extravasation. ${ }^{9}$ We therefore strived for additional visual control of cement distribution and, in case of intraspinal cement extravasation, for direct access to the spinal canal to remove leaked cement. Moreover, we were able to achieve gross-total resection of the intravertebral tumor, thereby creating a cavity for an adequate volume of bone cement.

We believe that minimally invasive resection and vertebroplasty via a tubular retractor overcome the above-mentioned issues. Accordingly, surgery was performed without complications. The extended course, in fact, showed segmental deviation due to progressive tumor growth and bony destruction, but the patient complained only about minor neck pain. Therefore, we suggest our technique for selected palliative tumor patients with a limited life expectancy to preserve the mobility of the head, gain stability, and achieve prompt and permanent pain reduction. In patients with long-term life expectancy, vertebroplasty alone may not lead to sufficient segmental stability; hence, occipitocervical fusion should be considered.

\section{References}

1. Anselmetti GC, Manca A, Montemurro F, Tutton S, Chiara $\mathrm{G}$, Battistella M, et al: Vertebroplasty using transoral approach in painful malignant involvement of the second cervical vertebra (C2): a single-institution series of 25 patients. Pain Physician 15:35-42, 2012

2. Chuang HC, Lee HC, Cho DY: Intracranial malignant meningioma with multiple spinal metastases-a case report and literature review: case report. Spine (Phila Pa 1976) 31:E1006-E1010, 2006

3. Cianfoni A, Distefano D, Chin SH, Varma AK, Rumboldt Z, Bonaldi G: Percutaneous cement augmentation of a lytic lesion of $\mathrm{C} 1$ via posterolateral approach under CT guidance. Spine J 12:500-506, 2012

4. Clarençon F, Cormier E, Pascal-Moussellard H, Maldent JB, Pichon S, Le Jean L, et al: Transoral approach for percutane- 
ous vertebroplasty in the treatment of osteolytic tumor lesions of the lateral mass of the atlas: feasibility and initial experience in 2 patients. Spine (Phila Pa 1976) 38:E193-E197, 2013

5. Enam SA, Abdulrauf S, Mehta B, Malik GM, Mahmood A: Metastasis in meningioma. Acta Neurochir (Wien) 138:1172-1178, 1996

6. Eom KS, Kim HS, Kim TY, Kim JM: Intraventricular malignant meningioma with CSF-Disseminated spinal metastasis: case report and literature review. J Korean Neurosurg Soc 45:256-259, 2009

7. Guo WH, Meng MB, You X, Luo Y, Li J, Qiu M, et al: CTguided percutaneous vertebroplasty of the upper cervical spine via a translateral approach. Pain Physician 15:E733E741, 2012

8. Huegli RW, Schaeren S, Jacob AL, Martin JB, Wetzel SG: Percutaneous cervical vertebroplasty in a multifunctional image-guided therapy suite: hybrid lateral approach to $\mathrm{C} 1$ and $\mathrm{C} 4$ under $\mathrm{CT}$ and fluoroscopic guidance. Cardiovasc Intervent Radiol 28:649-652, 2005

9. Klingler JH, Sircar R, Deininger MH, Scheiwe C, Kogias E, Hubbe U: Vesselplasty: a new minimally invasive approach to treat pathological vertebral fractures in selected tumor patients-preliminary results. Rofo 185:340-350, 2013

10. Klingler JH, Sircar R, Hubbe U: [Cervical vertebroplastypercutaneous technique of the anterior approach.] Rofo 185:1202-1203, 2013 (Ger)

11. Kuroda H, Kashimura H, Ogasawara K, Sugawara A, Sasoh $\mathrm{M}$, Arai H, et al: Malignant intracranial meningioma with spinal metastasis-case report. Neurol Med Chir (Tokyo) 49:258-261, 2009

12. Lee TT, Landy HJ: Spinal metastases of malignant intracranial meningioma. Surg Neurol 50:437-441, 1998

13. Louis DN, Ohgaki H, Wiestler OD, Cavenee WK, Burger PC,
Jouvet A, et al: The 2007 WHO classification of tumours of the central nervous system. Acta Neuropathol 114:97-109, 2007

14. Modha A, Gutin PH: Diagnosis and treatment of atypical and anaplastic meningiomas: a review. Neurosurgery 57:538-50, 2005

15. Perry A, Scheithauer BW, Stafford SL, Lohse CM, Wollan PC: "Malignancy" in meningiomas: a clinicopathologic study of 116 patients, with grading implications. Cancer 85:2046-2056, 1999

16. Rosenberg LA, Prayson RA, Lee J, Reddy C, Chao ST, Barnett GH, et al: Long-term experience with World Health Organization grade III (malignant) meningiomas at a single institution. Int J Radiat Oncol Biol Phys 74:427-432, 2009

17. Wetzel SG, Martin JB, Somon T, Wilhelm K, Rufenacht DA: Painful osteolytic metastasis of the atlas: treatment with percutaneous vertebroplasty. Spine (Phila Pa 1976) 27:E493$\mathrm{E} 495,2002$

\section{Author Contributions}

Conception and design: Klingler. Acquisition of data: Klingler. Analysis and interpretation of data: Klingler. Drafting the article: Klingler. Critically revising the article: all authors. Reviewed submitted version of manuscript: all authors. Approved the final version of the manuscript on behalf of all authors: Klingler. Administrative/technical/material support: Krüger, Brendecke.

\section{Correspondence}

Jan-Helge Klingler, Department of Neurosurgery, Freiburg University Medical Center, Breisacher Str. 64, Freiburg D-79106, Germany.email: jan-helge.klingler@uniklinik-freiburg.de. 